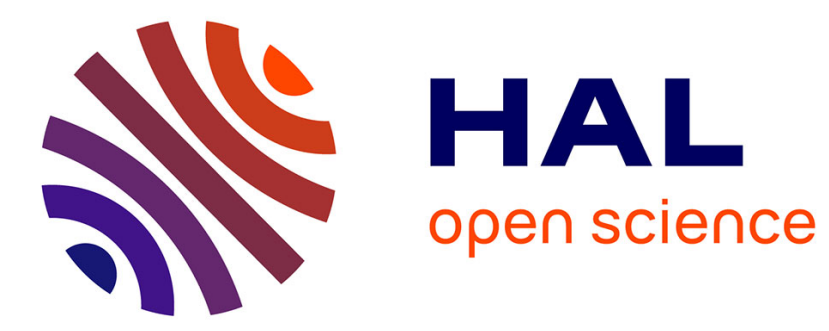

\title{
On the most imbalanced orientation of a graph
}

\author{
Walid Ben-Ameur, Antoine Glorieux, José Neto
}

\section{To cite this version:}

Walid Ben-Ameur, Antoine Glorieux, José Neto. On the most imbalanced orientation of a graph. COCOON 2015 : 21st International Conference on Computing and Combinatorics, Aug 2015, Beijing, China. pp.16 - 29, 10.1007/978-3-319-21398-9_2 . hal-01497825

\section{HAL Id: hal-01497825 \\ https://hal.science/hal-01497825}

Submitted on 29 Mar 2017

HAL is a multi-disciplinary open access archive for the deposit and dissemination of scientific research documents, whether they are published or not. The documents may come from teaching and research institutions in France or abroad, or from public or private research centers.
L'archive ouverte pluridisciplinaire HAL, est destinée au dépôt et à la diffusion de documents scientifiques de niveau recherche, publiés ou non, émanant des établissements d'enseignement et de recherche français ou étrangers, des laboratoires publics ou privés. 


\title{
On the most imbalanced orientation of a graph
}

\author{
Walid Ben-Ameur, Antoine Glorieux, and José Neto \\ Institut Mines-Télécom, Télécom SudParis, CNRS Samovar UMR 5157 \\ 9 Rue Charles Fourier, 91011 Evry Cedex, France \\ walid.benameur@telecom-sudparis.eu, antoine.glorieux@telecom-sudparis.eu, \\ jose.neto@telecom-sudparis.eu
}

\begin{abstract}
We study the problem of orienting the edges of a graph such that the minimum over all the vertices of the absolute difference between the outdegree and the indegree of a vertex is maximized. We call this minimum the imbalance of the orientation, i.e. the higher it gets, the more imbalanced the orientation is. We study this problem denoted by MAXIM. We first present different characterizations of the graphs for which the optimal objective value of MАХIM is zero. Next we show that it is generally NP-complete and cannot be approximated within a ratio of $\frac{1}{2}+\varepsilon$ for any constant $\varepsilon>0$ in polynomial time unless $P=N P$ even if the minimum degree of the graph $\delta$ equals 2 . Finally we describe a polynomial-time approximation algorithm whose ratio is almost equal to $\frac{1}{2}$.
\end{abstract}

\section{Introduction and notation}

Let $G=(V, E)$ be an undirected simple graph, we denote by $\delta_{G}$ the minimum degree of the vertices of $G$. An orientation $\Lambda$ of $G$ is an assignment of a direction to each undirected edge $\{u v\}$ in $E$, i.e. any function on $E$ of the form $\Lambda(\{u v\}) \in\{u v, v u\}$, $\forall\{u v\} \in E$. For each vertex $v$ of $\mathrm{G}$ we denote by $d_{G}(v)$ or $d(v)$ the unoriented degree of $v$ in $G$ and by $d_{\Lambda}^{+}(v)$ or $d^{+}(v)$ (resp. $d_{\Lambda}^{-}(v)$ or $d^{-}(v)$ ) the outdegree (resp. indegree) of $v$ in $G$ w.r.t. $\Lambda$. Graph orientation is a well studied area in graph theory and combinatorial optimization and thus a large variety of constrained orientations as well as objective functions have been considered so far.

Among those arise the popular degree-constrained orientation problems: in 1976, Frank \& Gyárfás [12] gave a simple characterization of the existence of an orientation such that the outdgree of every vertex is between a lower and an upper bound given for each vertex. Asahiro et al. in [1,2,3] proved the NP-hardness of the weighted version of the problem where the maximum outdegree is minimized, gave some inapproximability results, and studied similar problems for different classes of graphs. Chrobak \& Eppstein proved that for every planar graph a 3-bounded outdegree orientation and a 5-bounded outdegree acyclic orientation can be constructed in linear time [6].

Other problems involving other criterion on the orientation have been studied such as acyclicity, diameter or connectivity. Robbins' theorem (1939) for example states that the graphs that have strong orientations are exactly the 2-edge-connected graphs [18] and later (1985), Chung et al. provided a linear time algorithm for checking whether a graph has such an orientation and finding one if it does [7]. Then in 1960, NashWilliams generalized Robbin's theorem showing that an undirected graph has a $k$-arcconnected orientation if and only if it is $2 k$-edge-connected [17]. The problem called 
oriented diameter that consists in finding a strongly connected orientation with minimum diameter was introduced in 1978 by Chv́atal \& Thomassen: they proved that the problem is NP-hard for general graphs [8]. It was then proven to be NP-hard even if the graph is restricted to a subset of chordal graphs by Fomin et al. (2004) who gave also approximability and inapproximability results [10].

For an orientation $\Lambda$ of $G=(V, E)$ and a vertex $v$ we call $\left|d_{\Lambda}^{+}(v)-d_{\Lambda}^{-}(v)\right|$ the imbalance of $v$ in $G$ w.r.t $\Lambda$ and thus we call $\min _{v \in V}\left|d_{\Lambda}^{+}(v)-d_{\Lambda}^{-}(v)\right|$ the imbalance of $\Lambda$. Biedl et al. studied the problem of finding an acyclic orientation of unweighted graphs minimizing the imbalance of each vertex: they proved that it is solvable in polynomial time for graphs with maximum degree at most three but NP-complete generally and for bipartite graphs with maximum degree six and gave a $\frac{13}{8}$-approximation algorithm [5]. Then Kára et al. closed the gap proving the NP-completeness for graphs with maximum degree four. Furthermore, they proved that the problem remains NP-complete for planar graphs with maximum degree four and for 5-regular graphs [14].

Landau's famous theorem [15] gives a condition for a sequence of non-negative integers to be the score sequence or outdegree sequence of some tournament (i.e. oriented complete graph) and later, Harary \& Moser characterized score sequences of strongly connected tournaments [13]. Analogous results for the "imbalance sequences" of directed graphs are were given by Mubayi et al. [16]. In 1962, Ford \& Fulkerson characterized the mixed graphs (i.e. partially oriented graphs) which orientation can be completed in a eulerian orientation, that is to say, an orientation for which the imbalance of each vertex equals zero [11]. Many other results related to orientation have been proposed. Some of them are reviewed in [4].

Let us denote by $\vec{O}(G)$ the set of all the orientations of $G$, we consider the problem of finding an orientation with maximized imbalance:

$$
(\operatorname{Maxim}) \operatorname{Maxim}(G)=\max _{\Lambda \in \vec{O}(G)} \min _{v \in V}\left|d_{\Lambda}^{+}(v)-d_{\Lambda}^{-}(v)\right|
$$

and we call $\operatorname{MAXIM}(G)$ the value of MAXIM for $G$. The minimum degree $\delta_{G}$ of a graph $G$ is a trivial upper bound for $\operatorname{MAXIM}(G)$.

The rest of this paper is organized as follows. In the first section, we give several characterizations of the the graphs verifying $\operatorname{MAxIm}(G)=0$. In section 2, we will show that MAXIM is generally NP-complete even for graphs with minimum degree 2 and inapproximable within a ratio $\frac{1}{2}+\varepsilon$ for any constant $\varepsilon>0$ and then will give an approximation algorithm whose ratio is almost equal to $\frac{1}{2}$. Since the value of MAXIM for a graph is the minimum of the values of MAXIM on its connected component, from here on in, all the graphs we consider are assumed to be connected. For any graph $G$ we will use the notations $V(G)$ and $E(G)$ to refer to the set of vertices of $G$ and the set of edges of $G$ respectively.

\section{Characterizing the graphs for which $\operatorname{MAXIM}(G)=0$}

Now we ask ourselves which are the graphs verifying $\operatorname{MAxIm}(G)=0$. We will start by unveiling several necessary conditions and properties of such graphs. First we can show 
that concerning such a graph, we can find an orientation satisfying several additional properties.

Proposition 1 Let $G$ be a graph such that $\operatorname{MAxIm}(G)=0$ and $u \in V$. Then there exists an orientation $\Lambda \in \vec{O}(G)$ such that $u$ is the only vertex of $G$ with imbalance equal to zero w.r.t. $\Lambda$.

Proof. Let $\Lambda \in \vec{O}(G)$ be an orientation minimizing $\left|\left\{v \in V /\left|d_{\Lambda}^{+}(v)-d_{\Lambda}^{-}(v)\right|=0\right\}\right|$. We suppose that $\left|\left\{v \in V /\left|d_{\Lambda}^{+}(v)-d_{\Lambda}^{-}(v)\right|=0\right\}\right| \geq 2$. We choose two distinct vertices $v$ and $w$ in $\left\{v \in V /\left|d_{\Lambda}^{+}(v)-d_{\Lambda}^{-}(v)\right|=0\right\}$ and a path $p=\left(v=u_{0}, \cdots, u_{n}=w\right)$ between $v$ and $w$. If we switch the orientation of the edge $\left\{u_{0} u_{1}\right\}$, then the imbalance of $u_{0}$ becomes positive and necessarily the imbalance of $u_{1}$ becomes zero otherwise the resulting orientation would contradict the minimality of $\Lambda$. Using the same reasoning, if we switch the orientation of all the edges $\left\{u_{0} u_{1}\right\}, \cdots,\left\{u_{n-2} u_{n-1}\right\}$, we obtain an orientation where both $u_{n-1}$ and $u_{n}$ have an imbalance equal to zero while the imbalance is positive on all the vertices $u_{0}, \cdots, u_{n-2}$ and unchanged on all other vertices. So now if we switch the orientation of the edge $\left\{u_{n-1} u_{n}\right\}$ as well, then the resulting orientation contradicts the minimality of $\Lambda$. Hence, $\left|\left\{v \in V /\left|d_{\Lambda}^{+}(v)-d_{\Lambda}^{-}(v)\right|=0\right\}\right|=1$.

Now let $v$ be this unique vertex of $G$ such that $\left|d_{\Lambda}^{+}(v)-d_{\Lambda}^{-}(v)\right|=0$. Let $u \neq v$ be an arbitrary vertex and let $p=\left(v=u_{0}, \cdots, u_{n}=u\right)$ be a path between $v$ and $u$. By switching the orientation of all the edges $\left\{u_{0} u_{1}\right\}, \cdots,\left\{u_{n-2} u_{n-1}\right\}$, we obtain an orientation $\Lambda^{\prime}$ where $u$ has an imbalance equal to zero while the imbalance is positive for $u_{0}$ and unchanged on all other vertices.

This yields the following necessary condition: if $G$ is a graph such that $\operatorname{MAxIM}(G)=$ 0 , then $G$ is eulerian. For let $u \in V$, we know there exists $\Lambda \in \vec{O}(G)$ such that $\{v \in$ $\left.V /\left|d_{\Lambda}^{+}(v)-d_{\Lambda}^{-}(v)\right|=0\right\}=\{u\}$. Then $d_{\Lambda}^{+}(u)=d_{\Lambda}^{-}(u)$, hence $d(u)=d_{\Lambda}^{+}(u)+d_{\Lambda}^{-}(u)=$ $2 d_{\Lambda}^{+}(u)$ is even. The following lemma about eulerian graphs will prove useful for the proof of our characterization.

Lemma 2 If $G$ is an eulerian graph, then there exists an elementary cycle (hereafter just called cycle) $C$ of $G$ such that $G-E(C)$ has at most one connected component that is not an isolated vertex.

Proof. Being $G$ eulerian and connected, it can be decomposed into edge-disjoint cycles that we can order $C_{1}, \cdots, C_{n}$ according to the following condition: $\cup_{k=1}^{i} C_{i}$ is connected, $\forall i \in \llbracket 1, n \rrbracket$. Then $C_{n}$ is the cycle we are looking for.

Now let us define a certain family of graphs which will prove to be exactly the graphs for which the optimal objective value of MAXIM is zero. Intuitively they are the graphs for which every block is an odd cycle.

Theorem 3 We define the class of graphs $\mathscr{C}^{\text {odd }}$ as follows: a simple graph $G$ is in $\mathscr{C}^{\text {odd }}$ if there exists $C_{1}, \cdots, C_{n}$ odd cycles $(n \geq 1)$ such that:

- $\cup_{i=1}^{n} C_{i}=G$,

- $\left|V\left(\cup_{k=1}^{i-1} C_{k}\right) \cap V\left(C_{i}\right)\right|=1, \forall i \in \llbracket 2, n \rrbracket$.

Then for any simple graph $G, \operatorname{MAxIm}(G)=0$ if and only if $G \in \mathscr{C}^{\text {odd }}$. 
Proof. $\quad \Leftarrow \Leftarrow$ We will work by induction on the number of cycles $n$ contained in the graph. Nothing is required for these cycles except that they must be elementary. If $n=1$, then our graph is an odd cycle which implies $\operatorname{MAXIM}(G)=0$. Let $n \geq 2$, we assume that all graphs of $\mathscr{C}^{\text {odd }}$ with $k \leq n-1$ cycles verify $\operatorname{MAxIm}(G)=0$. Let $G \in \mathscr{C}^{\text {odd }}$ with $n$ cycles $C_{1}, \cdots, C_{n}$ as in (1). Suppose there exists $\Lambda \in \vec{O}(G)$ with strictly positive imbalance. Let us call $G^{\prime}=\cup_{i=1}^{n-1} C_{i}$ the graph obtained from $G$ after removing $C_{n}$ and let us take a look at $\Lambda_{\mid E\left(G^{\prime}\right)}$ the orientation of the edges of $G^{\prime}$ obtained from $\Lambda$ as its restriction on $E\left(G^{\prime}\right)$. As $G^{\prime}$ is a graph of $n-1$ cycles in $\mathscr{C}^{\text {odd }}$, our inductive hypothesis implies that we have a vertex $u \in V\left(G^{\prime}\right)$ such that $\left|d_{\Lambda_{\mid E\left(G^{\prime}\right)}^{+}}^{+}(u)-d_{\Lambda_{\mid E\left(G^{\prime}\right)}^{-}}(u)\right|=0$. Necessarily, $u=V\left(G^{\prime}\right) \cap V\left(C_{n}\right)$. Thus $\mid d_{\Lambda}^{+}(u)-$ $d_{\Lambda}^{-}(u)|=| d_{\Lambda_{\mid E\left(C_{n}\right)}^{+}}^{+}(u)-d_{\Lambda_{\mid E\left(C_{n}\right)}^{-}}(u) \mid>0$ implying that $\operatorname{MAXIM}\left(C_{n}\right)>0$ which is absurd because $C_{n}$ is an odd cycle.

- $\Rightarrow$ Since $\operatorname{Maxim}(G)=0$, we know that $G$ is eulerian. We will again work by induction on the number of cycles $n$. If $n=1$, then our graph is eulerian with a unique cycle, hence it is a cycle. Now as $\operatorname{MaxIm}(G)=0$, necessarily it is an odd cycle and is therefore in $\mathscr{C}^{\text {odd }}$. Let $n \geq 2$, we assume that all graphs with $k \leq n-1$ cycles verifying $\operatorname{MAXIM}(G)=0$ are in $\mathscr{C}^{\text {odd }}$. Let $G$ be a graph with $n$ cycles such that $\operatorname{MaxIm}(G)=0$. Thanks to Lemma 2, there exists an cycle $C$ of $G$ such that $G-E(C)$ has at most one connected component $G^{\prime}$ that is not an isolated vertex.

Suppose that $\operatorname{MAxIm}\left(G^{\prime}\right)>0$, let $\Lambda \in \vec{O}\left(G^{\prime}\right)$ with strictly positive imbalance. Let $u_{0} \in V\left(G^{\prime}\right) \cap V(C)$, we name the vertices of $C$ as follows: $u_{0}, u_{1}, \cdots, u_{k}=u_{0}$. Without loss of generality, we can assume that $d_{\Lambda}^{+}\left(u_{0}\right)-d_{\Lambda}^{-}\left(u_{0}\right)>0$; if it was not the case, replace $\Lambda$ by its reverse. We complete $\Lambda$ in an orientation of $G$ by orienting the edges of $C$ : we orient $u_{0} u_{1}$ from $u_{0}$ to $u_{1}$ and go on as follows:

$$
\forall i \in \llbracket 1, k-1 \rrbracket, \begin{cases}\text { if } u_{i} \in V\left(G^{\prime}\right), & \text { we orient }\left\{u_{i} u_{i+1}\right\} \text { as }\left\{u_{i-1} u_{i}\right\}, \\ \text { otherwise, } & \text { we orient }\left\{u_{i} u_{i+1}\right\} \text { as }\left\{u_{i} u_{i-1}\right\} .\end{cases}
$$

Where orienting an edge $\{a b\}$ as another edge $\{c d\}$ means orienting it from $a$ to $b$ if $\{c d\}$ was oriented from $c$ to $d$ and from $b$ to $a$ otherwise. Let us have a look at the resulting orientation $\Lambda^{\prime}$ (cf Figure 1): when completing $\Lambda$ in $\Lambda^{\prime}$, the imbalance of the vertices in $V\left(G^{\prime}\right) \backslash\left\{u_{0}\right\}$ was left unchanged, the imbalance of the vertices in $V(C) \backslash V\left(G^{\prime}\right)$ equals 2 and the imbalance of $u_{0}$ was either left unchanged or augmented by two. Hence $\Lambda^{\prime}$ has strictly positive imbalance which contraditcts $\operatorname{MaxIm}(G)=0$, therefore, $\operatorname{MaxIm}\left(G^{\prime}\right)=0$.

Suppose $\left|V\left(G^{\prime}\right) \cap V(C)\right| \geq 2$ and let $u$ and $v$ be 2 distinct vertices in $\left.V\left(G^{\prime}\right) \cap V(C)\right)$ such that $u \neq v$. Thanks to proposition 1, we know that there exists an orientation $\Lambda \in \vec{O}\left(G^{\prime}\right)$ such that $\left\{w \in V /\left|d_{\Lambda}^{+}(w)-d_{\Lambda}^{-}(w)\right|=0\right\}=\{v\}$ and without loss of generality, $d_{\Lambda}^{+}(u)-d_{\Lambda}^{-}(u)>0$. We name the vertices of $C$ as follows: $u=u_{0} u_{1} \cdots u_{k}=u_{0}, v=u_{l}$ and we complete $\Lambda$ in an orientation of $G$ by orienting the edges of $C$ : we orient $\left\{u_{0} u_{1}\right\}$ from $u_{0}$ and $u_{1}$ and go on as follows:

$$
\forall i \in \llbracket 1, k-1 \rrbracket \backslash\{l\}, \begin{cases}\text { if } u_{i} \in V\left(G^{\prime}\right), & \text { we orient }\left\{u_{i} u_{i+1}\right\} \text { as }\left\{u_{i-1} u_{i}\right\}, \\ \text { otherwise, } & \text { we orient }\left\{u_{i} u_{i+1}\right\} \text { as }\left\{u_{i} u_{i-1}\right\} .\end{cases}
$$


And we orient $\left\{u_{l} u_{l+1}\right\}$ as $\left\{u_{l} u_{l-1}\right\}$. In the resulting orientation $\Lambda^{\prime}$, the imbalance of the vertices in $V\left(G^{\prime}\right) \backslash\{u, v\}$ was left unchanged, the imbalance of the vertices in $V(C) \backslash V\left(G^{\prime}\right)$ equals 2 , the imbalance of $v$ was augmented by two and the imbalance of $u$ was either left unchanged or augmented by two. Hence $\Lambda^{\prime}$ contradicts $\operatorname{MaxIm}(G)=0$, therefore, $\left|V\left(G^{\prime}\right) \cap V(C)\right|=1$.

Suppose $C$ is even. We call $u \in V\left(G^{\prime}\right)$ such that $V\left(G^{\prime}\right) \cap V(C)=\{u\}$, and $\Lambda \in$ $\vec{O}\left(G^{\prime}\right)$ such that $\left\{v \in V /\left|d_{\Lambda}^{+}(v)-d_{\Lambda}^{-}(v)\right|=0\right\}=\{u\}$. We name the vertices of $C$ as follows: $u=u_{0} u_{1} \cdots u_{k}=u_{0}$ and we complete $\Lambda$ in an orientation of $G$ by orienting the edges of $C$ : we orient $\left\{u_{0} u_{1}\right\}$ from $u_{0}$ to $u_{1}$ and $\left\{u_{i} u_{i+1}\right\}$ as $\left\{u_{i} u_{i-1}\right\}, \forall i \in$ $\llbracket 1, k-1 \rrbracket$. In the resulting orientation $\Lambda^{\prime}$, the imbalance of the vertices in $V\left(G^{\prime}\right) \backslash\{u\}$ was left unchanged, the imbalance of the vertices in $V(C) \backslash V\left(G^{\prime}\right)$ equals 2 and, $C$ being even, the imbalance of $u$ was augmented by two. Hence $\Lambda^{\prime}$ contradicts $\operatorname{MAXIm}(G)=0$, therefore, $C$ is odd.

As $G^{\prime}$ is a graph with at most $n-1$ cycles verifying $\operatorname{MAxIm}(G)=0$, by induction hypothesis, there exist $C_{1}, \cdots, C_{n-1}$ odd cycles such that:

$\circ \cup_{i=1}^{n-1} C_{i}=G^{\prime}$

○ $\left|V\left(\cup_{k=1}^{i-1} C_{k}\right) \cap V\left(C_{i}\right)\right|=1, \forall i \in \llbracket 2, n-1 \rrbracket$.

Adding the odd cycle $C_{n}=C$, we directly obtain that $G \in \mathscr{C}^{\text {odd }}$.

Fig. 1: The vertices of $C$ in $G^{\prime}$ are left unchanged imbalance-wise, the other vertices of $C$ are set to 2 and in the end $\left|d_{\Lambda^{\prime}}^{+}\left(u_{0}\right)-d_{\Lambda^{\prime}}^{-}\left(u_{0}\right)\right| \geq\left|d_{\Lambda}^{+}\left(u_{0}\right)-d_{\Lambda}^{-}\left(u_{0}\right)\right|>0$

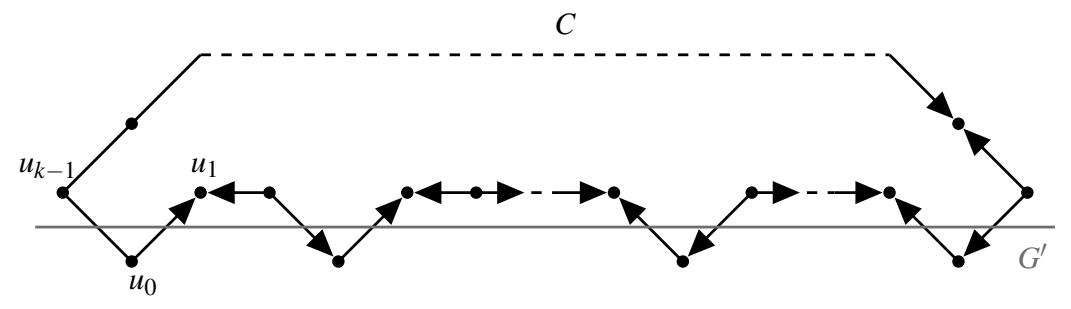

Now in order to widen our perception of those graphs, let us show another characterization.

Theorem 4 For every simple graph $G$,

$$
G \in \mathscr{C}^{\text {odd }} \Leftrightarrow G \text { is eulerian with no even cycle }
$$

Proof. $\quad-\Rightarrow$ By construction, every graph in $\mathscr{C}^{\text {odd }}$ is eulerian with no even cycle. 
Fig. 2: The vertices of $C$ in $G^{\prime}$ are left unchanged imbalance-wise except for $v$ which is set to 2, like the other vertices of $C$ and in the end $\left|d_{\Lambda^{\prime}}^{+}\left(u_{0}\right)-d_{\Lambda^{\prime}}^{-}\left(u_{0}\right)\right| \geq\left|d_{\Lambda}^{+}\left(u_{0}\right)-d_{\Lambda}^{-}\left(u_{0}\right)\right|>0$

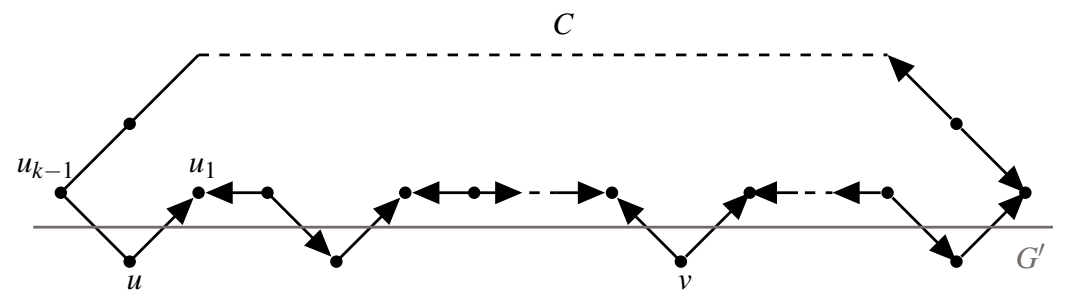

- $\Leftarrow$ We will once again work by induction on the number of cycles $n$.

If $n=1$, then our graph is eulerian with a unique odd cycle, hence it is an odd cycle and is therefore in $\mathscr{C}^{\text {odd }}$.

Let $n \geq 2$, we assume that all eulerian graphs with no even cycle and $k \leq n-1$ odd cycles are in $\mathscr{C}^{\text {odd }}$. Let $G$ be a graph with no even cycle and $n$ odd cycles. Thanks to Lemma 2, there exists an odd cycle $C$ of $G$ such that $G-E(C)$ has only one connected component $G^{\prime}$ that is not an isolated vertex. As $G^{\prime}$ is eulerian and even-cycle-free with $n-1$ odd cycles, by induction hypothesis, $G^{\prime} \in \mathscr{C}^{\text {odd }}$, hence there exist $C_{1}, \cdots, C_{n-1}$ odd cycles such that:

○ $\cup_{i=1}^{n-1} C_{i}=G^{\prime}$

○ $\left|V\left(\cup_{k=1}^{i-1} C_{k}\right) \cap V\left(C_{i}\right)\right|=1, \forall i \in \llbracket 2, n-1 \rrbracket$.

Suppose there exist $u$ and $v(u \neq v)$ belonging to $V\left(\cup_{k=1}^{n-1} C_{k}\right) \cap V(C)$. Since $G^{\prime}$ is connected, let $p$ be an elementary path in $G^{\prime}$ between $u$ and $v$. We can assume that $u$ and $v$ are the only vertices of $C$ contained in $p$, otherwise we could replace $v$ by the first vertex of $C$ encountered when travelling on $p$ from $u$. $C$ defines two other vertex-disjoint paths between $u$ and $v$ : one even that we will call $p_{\text {even }}$ and one odd that we will call $p_{\text {odd }} \cdot p$ being vertex disjoint with either $p_{\text {even }}$ or $p_{\text {odd }}$, by concatenating it with the one corresponding to its parity, we obtain an even cycle of $G$, contradicting our hypothesis on $G$. This yields that $\left|V(C) \cap V\left(G^{\prime}\right)\right|=1$. From that we can conclude

$$
\begin{array}{ll}
\circ & \cup{ }_{i=1}^{n} C_{i}=G, \\
\circ & \left|V\left(\cup_{k=1}^{i-1} C_{k}\right) \cap V\left(C_{i}\right)\right|=1, \forall i \in \llbracket 2, n \rrbracket .
\end{array}
$$

Hence $G \in \mathscr{C}^{\text {odd }}$.

\section{Complexity, inapproximability and approximability}

In this section we will prove the NP-completeness and inapproximability of our problem and give an approximation algorithm based on the special case of bipartite graphs.

Concerning the complexity of MAXIM, we will show that the problem is NPcomplete. More precisely, that answering if $\operatorname{MAxIM}(G)$ equals 2 for a graph $G$ such 
that $\delta_{G}=2$ is NP-complete. For that purpose we will introduce a variant of the satisfiability problem that we will reduce to a MAXIM instance: the not-all-equal at most 3-SAT(3V).

Not-all-equal at most 3-SAT(3V) is a restriction of not-all-equal at most 3-SAT which is itself a restriction of 3-SAT known to be NP-complete [19] where each clause contains at most three literals and in each clause, not all the literals can be true. Since 2-SAT can be solved in polynomial time, we hereafter deal only with formulas having at least one three-literals clause. The added restriction of not-all-equal at most 3-SAT(3V) is that each variable (not literal) appears at most three times in a formula. The resulting problem is still NP-complete.

Lemma 5 The not-all-equal at most 3-SAT(3V) problem is NP-complete.

Proof. See Appendix A.

Now we will associate to a not-all-equal at most 3-SAT(3V) instance $\varphi$ with $n$ variables $\left\{x_{1}, \cdots, x_{n}\right\}$ and $m$ clauses $\left\{c_{1}, \cdots, c_{m}\right\}$ a graph $G_{\varphi}$ for which the value w.r.t. MAXIM will give the answer to whether $\varphi$ is satisfiable or not. If a variable $x_{i}$ occurs only in positive literals (resp. only in negative literals), it follows that a satisfying assignment of the variables of $\varphi$ must necssarily give the value TRUE (resp. FALSE) to $x_{i}$, therefore $x_{i}$ can be removed from $\varphi$ with conservation of the satisfiability. Thus, without loss of generality, we can assume that in any not-all-equal at most 3-SAT(3V) formula, every variable occurs at least once as a positive literal and at least once as a negative literal. $G_{\varphi}$ consists of gadgets that mimic the variables and the clauses of $\varphi$ and additional edges that connect them together:

- the gadget corresponding to a variable $x_{i}$ consists of two vertices labeled $x_{i}$ and $\neg x_{i}$ and one edge connecting them;

- the gadget corresponding to a two-literals clause $c_{j}=\left(l^{1} \vee l^{2}\right)$, where $l^{1}$ and $l^{2}$ are its literals, consists in two vertices labeled $a_{l^{1}}^{j}$ and $b_{l^{2}}^{j}$ corresponding to $l^{1}$ and $l^{2}$ respectively (the index " ${ }^{k}$ " of the vertices labels stands for the literal they represent, i.e. $x_{i}$ if $l^{k}$ is the variable $x_{i}$ and $\neg x_{i}$ if $l^{k}$ is the negation of the variable $x_{i}$ ) and one edge connecting them;

- the gadget corresponding to a three-literals clause gadget consists in six vertices and six edges. For a clause $c_{j}=\left(l^{1} \vee l^{2} \vee l^{3}\right)$, where $l^{1}, l^{2}$ and $l^{3}$ are its literals (the order is arbitrary), three vertices labeled $a_{l^{1}}^{j}, b_{l^{2}}^{j}$ and $b_{l^{3}}^{j}$ correspond to $l^{1}, l^{2}$ and $l^{3}$ respectively. Three additional vertices are labeled $u^{j}, v^{j}$ and $w^{j}$ and the gadgets' edges are $\left\{a_{l^{1}}^{j} u_{j}\right\},\left\{a_{l^{1}}^{j} v_{j}\right\},\left\{u_{j} w_{j}\right\},\left\{v_{j} w_{j}\right\},\left\{w_{j} b_{l^{2}}^{j}\right\}$ and $\left\{w_{j} b_{l^{3}}^{\prime j}\right\}$;

- $\forall i \in \llbracket 1, n \rrbracket$, the vertex labeled $x_{i}$ (resp. $\neg x_{i}$ ) is connected to all the vertices labeled $a_{x_{i}}^{j}, b_{x_{i}}^{j}$ or $b_{x_{i}}^{\prime j}\left(\right.$ resp. $a_{\neg x_{i}}^{j}, b_{\neg x_{i}}^{j}$ or $\left.b_{\neg x_{i}}^{\prime j}\right), \forall j \in \llbracket 1, m \rrbracket$.

As an example, for a formula

$$
\varphi=\left(x_{1} \vee \neg x_{2} \vee x_{3}\right) \wedge\left(\neg x_{1} \vee \neg x_{3} \vee x_{4}\right) \wedge\left(x_{1} \vee \neg x_{2} \vee x_{4}\right) \wedge\left(x_{2} \vee \neg x_{4}\right),
$$


Fig. 3: $G_{\varphi}$ for $\varphi=\left(x_{1} \vee \neg x_{2} \vee x_{3}\right) \wedge\left(\neg x_{1} \vee \neg x_{3} \vee x_{4}\right) \wedge\left(x_{1} \vee \neg x_{2} \vee x_{4}\right) \wedge\left(x_{2} \vee \neg x_{4}\right)$

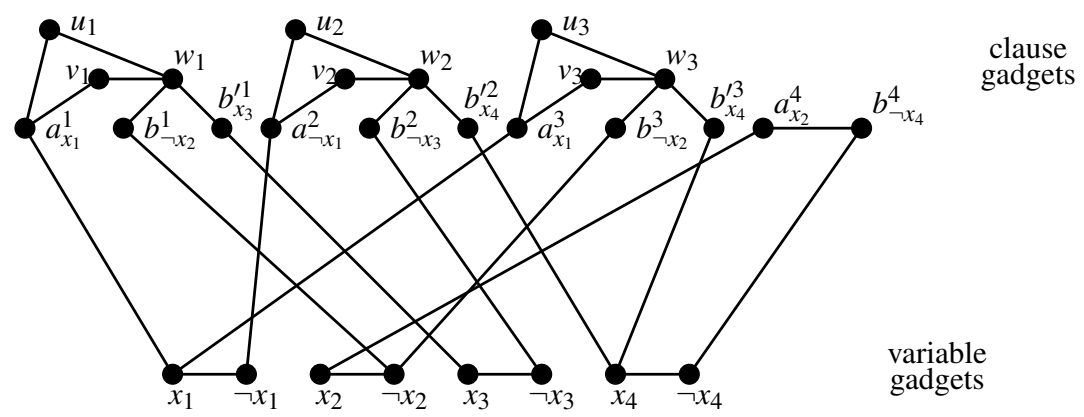

the corresponding graph $G_{\varphi}$ is represented in Figure 3.

Theorem 6 A not-all-equal at most 3-SAT(3V) formula $\varphi$ is satisfiable if and only if $\operatorname{MAxIm}\left(G_{\varphi}\right)=2$.

Proof. $\quad \Rightarrow$ Suppose $\varphi$ is satisfiable and let $v:\left\{x_{1}, \cdots, x_{n}\right\} \rightarrow\{$ TRUE, FALSE $\}$ be a satisfying assignment of $x_{1}, \cdots, x_{n}$. We know that $\delta_{G_{\varphi}}=2$ which yields $\operatorname{MAXIM}\left(G_{\varphi}\right) \leq$ 2. So let us build an orientation $\Lambda \in \vec{O}\left(G_{\varphi}\right)$ which imbalance is greater than or equal to 2. First, we assign an orientation to the edges of the variable gadget:

$$
\Lambda\left(\left\{x_{i} \neg x_{i}\right\}\right)= \begin{cases}x_{i} \neg x_{i} & \text { if } v\left(x_{i}\right)=\text { TRUE; } \\ \neg x_{i} x_{i} & \text { otherwise. }\end{cases}
$$

For example, for the formula $\varphi=\left(x_{1} \vee \neg x_{2} \vee x_{3}\right) \wedge\left(\neg x_{1} \vee \neg x_{3} \vee x_{4}\right) \wedge\left(x_{1} \vee \neg x_{2} \vee\right.$ $\left.x_{4}\right) \wedge\left(x_{2} \vee \neg x_{4}\right)$ satisfied by the assignment $v\left(x_{1}, x_{2}, x_{3}, x_{4}\right)=$ (FALSE, TRUE, TRUE, TRUE), the edges of the variable gadgets of graph $G_{\varphi}$ are oriented as in figure 4(a). Since each variable $x_{i}$ occurs at least once as a positive literal and at least once as a negative literal, $2 \leq d_{G_{\varphi}}\left(x_{i}\right) \leq 3$ and $2 \leq d_{G_{\varphi}}\left(\neg x_{i}\right) \leq 3, \forall i \in$ $\llbracket 1, n \rrbracket$. Then to ensure our objective on the imbalance of $\Lambda$, the orientation of the edges connecting vertex gadgets and clause gadgets must be such that $\forall i \in$ $\llbracket 1, n \rrbracket,\left|d_{\Lambda}^{+}\left(x_{i}\right)-d_{\Lambda}^{-}\left(x_{i}\right)\right|=d_{G_{\varphi}}\left(x_{i}\right)$ and $\left|d_{\Lambda}^{+}\left(\neg x_{i}\right)-d_{\Lambda}^{-}\left(\neg x_{i}\right)\right|=d_{G_{\varphi}}\left(\neg x_{i}\right)$. In other words, for $i \in \llbracket 1, n \rrbracket$, if $v\left(x_{i}\right)=$ TRUE (resp. $v\left(x_{i}\right)=$ FALSE), then the edges adjacent to the vertex $x_{i}$ are oriented from $x_{i}$ (resp. to $x_{i}$ ) and the edges adjacent to the vertex $\neg x_{i}$ are oriented to $\neg x_{i}$ (resp. from $\neg x_{i}$ ), e.g. Figure4(b).

So far, all the edges in the variables gadgets and the edges connecting the vertex gadgets and the clause gadgets have been oriented and the vertices in the variables gadgets have imbalance greater than or equal to 2 . In order to complete our orientation $\Lambda$ we have to orient the edges in the clause gadgets.

Let $c_{j}=\left(l^{1} \vee l^{2}\right)$ be a two-literals clause. Since $v$ satisfies $\varphi$, we know that exactly one of the two literals is true w.r.t. $v$. Which, according to the way we oriented edges so far, means that exactly one of $a_{l^{1}}^{j}$ and $b_{l^{2}}^{j}$ has one incoming arc from 
Fig. 4: $G_{\varphi}$ corresponding to $\varphi=\left(x_{1} \vee \neg x_{2} \vee x_{3}\right) \wedge\left(\neg x_{1} \vee \neg x_{3} \vee x_{4}\right) \wedge\left(x_{1} \vee \neg x_{2} \vee x_{4}\right) \wedge\left(x_{2} \vee \neg x_{4}\right)$ satisfied by $v\left(x_{1}, x_{2}, x_{3}, x_{4}\right)=($ FALSE, TRUE, TRUE, TRUE).

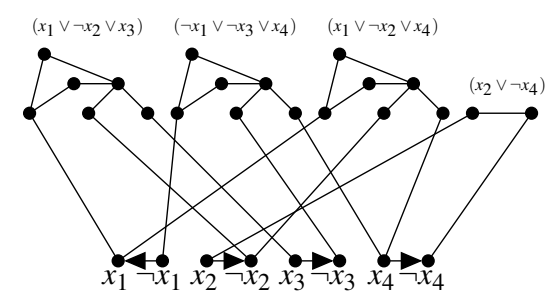

(a) orientation of the edges in the variable gadgets

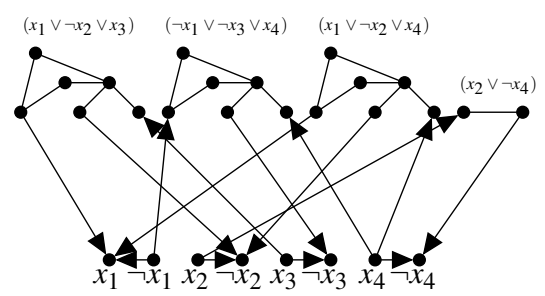

(b) orientation of the edges between the variable gadgets and the clause gadgets

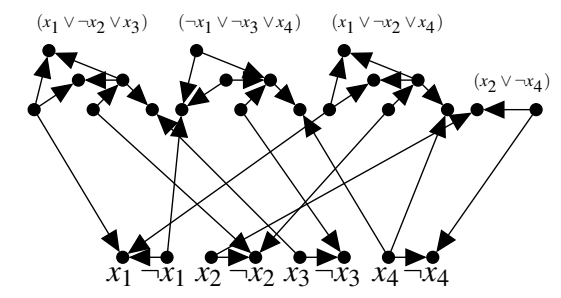

(c) orientation of the edges in the clause gadgets

a variable gadget and the other has one outgoing arc to a variable gadget. If $a_{l^{1}}^{j}$ is the one with the incoming arc from a variable gadget (meanings that $v\left(l^{1}\right)=$ TRUE), then we assign $\Lambda\left(\left\{a_{l^{1}}^{j} b_{l^{2}}^{j}\right\}\right)=\left(b_{l^{2}}^{j} a_{l^{1}}^{j}\right)$, otherwise the opposite. We obtain $\left|d_{\Lambda}^{+}\left(a_{l^{1}}^{j}\right)-d_{\Lambda}^{-}\left(a_{l^{1}}^{j}\right)\right|=\left|d_{\Lambda}^{+}\left(b_{l^{2}}^{j}\right)-d_{\Lambda}^{-}\left(b_{l^{2}}^{j}\right)\right|=2$.

Let $c_{j}=\left(l^{1} \vee l^{2} \vee l^{3}\right)$ (the order is identical to which was chosen to build the clause gadget, i.e. $d_{G_{\varphi}}\left(a_{l^{1}}^{j}\right)=3$ and $\left.d_{G_{\varphi}}\left(b_{l^{2}}^{j}\right)=d_{G_{\varphi}}\left(b_{l^{3}}^{j}\right)=2\right)$ be at three-literals clause. If the edge connecting $a_{l^{1}}^{j}$ to a variable gadget is oriented to $a_{l^{1}}^{j}$ (meanings that $v\left(l^{1}\right)=$ TRUE $)$, then we assign $\Lambda\left(\left\{a_{l^{1}}^{j} u_{j}\right\}\right)=\left(u_{j} a_{l^{1}}^{j}\right), \Lambda\left(\left\{a_{l^{1}}^{j} v_{j}\right\}\right)=\left(v_{j} a_{l^{1}}^{j}\right)$, $\Lambda\left(\left\{u_{j} w_{j}\right\}\right)=\left(u_{j} w_{j}\right)$ and $\Lambda\left(\left\{v_{j} w_{j}\right\}\right)=\left(v_{j} w_{j}\right)$. Since $v\left(l^{1}\right)=$ TRUE, either both $v\left(l^{2}\right)$ and $v\left(l^{3}\right)$ are FALSE or exactly one of $v\left(l^{2}\right)$ and $v\left(l^{3}\right)$ is TRUE and one is FALSE. If both are FALSE then $b_{l^{2}}^{j}$ and $b_{l^{3}}^{j}$ have an outgoing arc to a variable gadget. In that case, we orient $w_{j} b_{l^{2}}^{j}$ and $w_{j} b_{l^{3}}^{j}$ to $w_{j}$ and we obtain $\mid d_{\Lambda}^{+}\left(a_{l^{1}}^{j}\right)-$ $d_{\Lambda}^{-}\left(a_{l^{1}}^{j}\right)|=3,| d_{\Lambda}^{+}\left(b_{l^{2}}^{j}\right)-d_{\Lambda}^{-}\left(b_{l^{2}}^{j}\right)|=| d_{\Lambda}^{+}\left(\left(b_{l^{3}}^{\prime j}\right)-d_{\Lambda}^{-}\left(\left(b_{l^{3}}^{\prime j}\right)|=| d_{\Lambda}^{+}\left(u_{j}\right)-d_{\Lambda}^{-}\left(u_{j}\right) \mid=\right.\right.$ $\left|d_{\Lambda}^{+}\left(v_{j}\right)-d_{\Lambda}^{-}\left(v_{j}\right)\right|=2$ and $\left|d_{\Lambda}^{+}\left(w_{j}\right)-d_{\Lambda}^{-}\left(w_{j}\right)\right|=4$. If exactly one of $v\left(l^{2}\right)$ and $v\left(l^{3}\right)$ is TRUE and one is FALSE, then exactly one of $b_{l^{2}}^{j}$ and $b_{l^{3}}^{\prime j}$ has an incoming arc from a variable gadget and the other an outgoing arc to a variable gadget. If $b_{l^{2}}^{j}$ is the one with the incoming arc from a variable gadget (meanings that 
$v\left(l^{2}\right)=$ TRUE and $v\left(l^{3}\right)=$ FALSE $)$, then we assign $\Lambda\left(\left\{w_{j} b_{l^{2}}^{j}\right\}\right)=\left(w_{j} b_{l^{2}}^{j}\right)$ and $\Lambda\left(\left\{w_{j} b_{l^{3}}^{j}\right\}\right)=\left(b_{l^{3}}^{\prime j} w_{j}\right)$, otherwise the opposite. We obtain $\left|d_{\Lambda}^{+}\left(a_{l^{1}}^{j}\right)-d_{\Lambda}^{-}\left(a_{l^{1}}^{j}\right)\right|=3$ and $\left|d_{\Lambda}^{+}\left(b_{l^{2}}^{j}\right)-d_{\Lambda}^{-}\left(b_{l^{2}}^{j}\right)\right|=\left|d_{\Lambda}^{+}\left(b_{l^{3}}^{\prime j}\right)-d_{\Lambda}^{-}\left(b_{l^{3}}^{\prime j}\right)\right|=\left|d_{\Lambda}^{+}\left(u_{j}\right)-d_{\Lambda}^{-}\left(u_{j}\right)\right|=\mid d_{\Lambda}^{+}\left(v_{j}\right)-$ $d_{\Lambda}^{-}\left(v_{j}\right)|=| d_{\Lambda}^{+}\left(w_{j}\right)-d_{\Lambda}^{-}\left(w_{j}\right) \mid=2$.

If, on the other hand, the edge connecting $a_{l^{1}}^{j}$ to a variable gadget is oriented from $a_{l^{1}}^{j}$ (meanings that $v\left(l^{1}\right)=$ FALSE), then we assign $\Lambda\left(\left\{a_{l^{1}}^{j} u_{j}\right\}\right)=\left(a_{l^{1}}^{j} u_{j}\right)$, $\Lambda\left(\left\{a_{l^{1}}^{j} v_{j}\right\}\right)=\left(a_{l^{1}}^{j} v_{j}\right), \Lambda\left(\left\{u_{j} w_{j}\right\}\right)=\left(w_{j} u_{j}\right)$ and $\Lambda\left(\left\{v_{j} w_{j}\right\}\right)=\left(w_{j} v_{j}\right)$. By symmetry, we conclude in the same way that $\left|d_{\Lambda}^{+}\left(a_{l^{1}}^{j}\right)-d_{\Lambda}^{-}\left(a_{l^{1}}^{j}\right)\right|=3$ and $\mid d_{\Lambda}^{+}\left(b_{l^{2}}^{j}\right)-$ $d_{\Lambda}^{-}\left(b_{l^{2}}^{j}\right)|=| d_{\Lambda}^{+}\left(b_{l^{3}}^{\prime j}\right)-d_{\Lambda}^{-}\left(b_{l^{3}}^{\prime j}\right)|=| d_{\Lambda}^{+}\left(u_{j}\right)-d_{\Lambda}^{-}\left(u_{j}\right)|=| d_{\Lambda}^{+}\left(v_{j}\right)-d_{\Lambda}^{-}\left(v_{j}\right)|=| d_{\Lambda}^{+}\left(w_{j}\right)-$ $d_{\Lambda}^{-}\left(w_{j}\right) \mid=2$.

Consequently, the imbalance of the resulting orientation $\Lambda$ is greater than or equal to 2, e.g. Figure 4(c).

- $\Leftarrow$ Now we assume that $\operatorname{MAxIm}\left(G_{\varphi}\right)=2$, let $\Lambda \in \vec{O}\left(G_{\varphi}\right)$ with optimal imbalance. Since all the vertices in the variable gadgets have degree at most 3 , each vertex $x_{i}$ ( or $\neg x_{i}$ ) is necessarily adjacent to only incoming arcs or only outgoing arcs w.r.t. $\Lambda$. We will show that the assignment $v:\left\{x_{1}, \cdots, x_{n}\right\} \rightarrow\{$ TRUE, FALSE $\}$ of $x_{1}, \cdots, x_{n}$ defined by

$$
v\left(x_{i}\right)= \begin{cases}\text { TRUE } & \text { if } d_{\Lambda}^{+}\left(x_{i}\right)>d_{\Lambda}^{-}\left(x_{i}\right) ; \\ \text { FALSE } & \text { otherwise; }\end{cases}
$$

satisfies $\varphi$. Suppose $\varphi$ doesn't satisfy a clause $c_{j}, j \in \llbracket 1, m \rrbracket$. If $c_{j}$ is a two-literals clause $\left(l^{1} \vee l^{2}\right)$ then either $v\left(l^{1}\right)=v\left(l^{2}\right)=$ TRUE or $v\left(l^{1}\right)=v\left(l^{2}\right)=$ FALSE, i.e. either both $a_{l^{1}}^{j}$ and $b_{l^{2}}^{j}$ have an incoming arc from a variable gadget or both have an outgoing arc to a variable gadget and in both cases, whichever is the orientation assigned to $a_{l^{1}}^{j} b_{l^{2}}^{j}$ by $\Lambda$, either $a_{l^{1}}^{j}$ or $b_{l^{2}}^{j}$ have a zero imbalance which contradicts our assumption. So $c_{j}$ is a three-literals clause $\left(l^{1} \vee l^{2} \vee l^{3}\right)$ (the order is identical to which was chosen to build the clause gadget, i.e. $d_{G_{\varphi}}\left(a_{l^{1}}^{j}\right)=3$ and $d_{G_{\varphi}}\left(b_{l^{2}}^{j}\right)=$ $\left.d_{G_{\varphi}}\left(b_{l^{3}}^{\prime j}\right)=2\right)$. Then either $v\left(l^{1}\right)=v\left(l^{2}\right)=v\left(l^{3}\right)=$ TRUE or $v\left(l^{1}\right)=v\left(l^{2}\right)=v\left(l^{3}\right)=$ FALSE, i.e. either all $a_{l^{1}}^{j}, b_{l^{2}}^{j}$ and $b_{l^{3}}^{j}$ have an incoming arc from a variable gadget or they all have an outgoing arc to a variable gadget. In the first case, it implies $\Lambda\left(\left\{a_{l^{1}}^{j} u_{j}\right\}\right)=\left(u_{j} a_{l^{1}}^{j}\right), \Lambda\left(\left\{a_{l^{1}}^{j} v_{j}\right\}\right)=\left(v_{j} a_{l^{1}}^{j}\right), \Lambda\left(\left\{u_{j} w_{j}\right\}\right)=\left(u_{j} w_{j}\right), \Lambda\left(\left\{v_{j} w_{j}\right\}\right)=$ $\left(v_{j} w_{j}\right), \Lambda\left(\left\{w_{j} b_{l^{2}}^{j}\right\}\right)=\left(w_{j} b_{l^{2}}^{j}\right)$ and $\Lambda\left(\left\{w_{j} b_{l^{3}}^{j^{j}}\right\}\right)=\left(w_{j} b_{l^{3}}^{\prime j}\right)$, and we obtain $\mid d_{\Lambda}^{+}\left(w_{j}\right)-$ $d_{\Lambda}^{-}\left(w_{j}\right) \mid=0$ which contradicts the optimality of $\Lambda$. Similarly, in the second case it implies that the orientations assigned to the edges of the clause gadgets are the opposite from the previous ones and we obtain the same contradiction.

So we can conclude that $v$ does satisfy $\varphi$.

Corollary 7 MAXIM is NP-complete and inapproximable within $\frac{1}{2}+\varepsilon$ where $\varepsilon \in \mathbb{R}_{+}^{*}$, unless $\mathrm{P}=\mathrm{NP}$.

Proof. Let $\varepsilon \in \mathbb{R}_{+}^{*}$, suppose that there existed a polynomial approximation algorithm giving val $\geq\left(\frac{1}{2}+\varepsilon\right) \operatorname{MAXIM}(G)$ for an input graph $G$. Let $\varphi$ be a not-all-equal at most 
3-SAT(3V) formula and $G_{\varphi}$ its associated graph. Since $G_{\varphi}$ contains at least one threeliterals clause gadget, we know that $G_{\varphi}$ contains an even cycle and $\delta_{G_{\varphi}}=2$. This leads to $\operatorname{MAxIm}\left(G_{\varphi}\right) \in\{1,2\}$ and since $\left(\frac{1}{2}+\varepsilon\right) \operatorname{MAxIm}\left(G_{\varphi}\right) \leq v a l \leq \operatorname{MAxIm}\left(G_{\varphi}\right)$, if the polynomial approximation algorithm returns a value less than or equal to 1 then

$$
\left(\frac{1}{2}+\varepsilon\right) \operatorname{MAxIm}\left(G_{\varphi}\right) \leq 1 \Rightarrow \operatorname{MAxIm}\left(G_{\varphi}\right)<2 \Rightarrow \operatorname{MAxIm}\left(G_{\varphi}\right)=1 ;
$$

and if it returns a value greater than 1 , then $\operatorname{MAxIm}\left(G_{\varphi}\right)$ is greater than 1 hence equal to 2 . In other words the polynomial approximation algorithm output answers whether $\varphi$ is satisfiable or not which is absurd unless $P=N P$.

Now we consider the case of bipartite graphs: if $G=\left(V 1 \sqcup V_{2}, E\right)$ is a bipartite graph, the orientation that consists in assigning to each edge in $E$ the orientation from its extremity in $V_{1}$ to its extremity in $V_{2}$ has an imbalance equal to $\delta_{G}$, i.e. optimal. This simple case permits us to obtain the following lower bound:

Theorem 8 For every graph $G$,

$$
\operatorname{Maxim}(G) \geq\left\lceil\frac{\delta_{G}}{2}\right\rceil-1 .
$$

Proof. Let $\left(V_{1}, V_{2}\right)$ be a partition of $V$ corresponding to a cut $C \subset E$ such that we have $|\delta(\{v\}) \cap C| \geq\left\lceil\frac{d(v)}{2}\right\rceil, \forall v \in V$. Such a cut exists: for example a maximum cardinality cut verifies this property, otherwise we could find a higher cardinality cut by switching a vertex $v \in V$ s.t. $|\delta(\{v\}) \cap C|<\left\lceil\frac{d(v)}{2}\right\rceil$ from $V_{1}$ to $V_{2}$ (or the contrary). Moreover, if we iterated this process starting from a random cut, we would converge in polynomial time time to a such a cut. Now we define $\Lambda \in \vec{O}(G)$ as follows. We begin by orienting all edges in $C$ from $V_{1}$ to $V_{2}$. Then for any $i \in\{1,2\}$, we orient the edges of the induced subgraph $G\left[V_{i}\right]$. We add a new vertex $v_{0}$ and an edge between $v_{0}$ and each vertex with an odd degree in $G\left[V_{i}\right]$ if it isn't eulerian and we consider a decomposition of its edges into edge-disjoint cycles. we orient each of these cycles as a directed cycle. Removing $v_{0}$ if necessary, the imbalance of each vertex in $G\left[V_{i}\right]$ is now in $\{-1,0,1\}$ which implies that $\forall v \in V$ we have $\left|d_{\Lambda}^{+}(v)-d_{\Lambda}^{-}(v)\right| \geq\left\lceil\frac{d(v)}{2}\right\rceil-1$, hence, $\operatorname{MAXIM}(G) \geq\left\lceil\frac{\delta_{G}}{2}\right\rceil-1$.

From the proof proposed above, it is easy to see that when $\delta_{G} \equiv 0[4]$ then $\operatorname{MAxIM}(G)$ $\geq \frac{\delta_{G}}{2}$ while $\operatorname{MAxIm}(G) \geq \frac{\delta_{G}-1}{2}$ when $\delta_{G}$ is odd and $\operatorname{MAXIM}(G) \geq \frac{\delta_{G}}{2}-1$ when $\delta_{G} \equiv 2[4]$. This leads to an approximation algorithm whose ratio is $\frac{1}{2}$ (resp. $\frac{1}{2}-\frac{1}{2 \delta}$, $\frac{1}{2}-\frac{1}{\delta}$ ) when $\delta_{G} \equiv 0[4]$, (resp. $\delta_{G}$ is odd, $\delta_{G} \equiv 2[4]$ ).

\section{Further research}

While computing the most imbalanced orientation of a graph is generally difficult, the problem turns out to be easy for cactus graphs. It may be the same for other graph classes, characterizing such graph classes would be interesting.

We are currently looking for efficient mathematical programming formulations to solve the problem for large size graphs. Details will follow in the extended version of the paper. One can also study the weighted version of the problem. 


\section{References}

1. Y.Asahiro, E.Miyano, H.Ono \& K.Zenmyo: 'Approximation algorithms for the graph orientation minimizing the maximum weighted outdegree', Proc. of the 3rd Int. Conf. on Algorithmic Aspects in Information and Management (AAIM2007), LNCS 4508, 167-177 (2007)

2. Y. Asahiro, E. Miyano \& H. Ono: 'Graph classes and the complexity of the graph orientation minimizing the maximum weighted outdegree', Proceedings of the fourteenth Computing: the Australasian Theory Symposium(CATS2008), Wollongong, NSW, Australia (2008)

3. Y. Asahiro, J. Jansson, E. Miyano \& H. Ono: 'Deegree constrained graph orientation: maximum satisfaction and minimum violation', WAOA 2013, LNCS 8447, 24-36 (2014)

4. J. Bang-Jensen \& G. Gutin: Orientations of graphs and digraphs in 'Digraphs: Theory, Algorithms and applications', Springer, 2nd edition, 417-472 (2009)

5. T. Biedl , T. Chan, Y. Ganjali, M. Hajiaghayi \& D. R. Wood: 'Balanced vertex-orderings of graphs', Discrete Applied Mathematics 48 (1), 27-48 (2005)

6. M. Chrobak \& D. Eppstein: 'Planar orientations with low out-degree and compaction of adjacency matrices', Theoretical Computer Sciences 86, 243-266 (1991)

7. F. Chung, M. Garey \& R. Tarjan: 'Strongly connected orientations of mixed multigraphs', Networks 15, 477-484 (1985)

8. V. Chv́atal \& C. Thomassen: 'Distances in orientation of graphs', Journal of Combinatorial Theory, series B 24, 61-75 (1978)

9. R. Diestel: 'Graph Theory, 4th edition', Springer (2010)

10. F. Fomin, M. Matamala \& I. Rapaport: 'Complexity of approximating the oriented diameter of chordal graphs', Journal of Graph Theory 45 (4), 255-269 (2004)

11. L.R. Ford \& D.R. Fulkerson: 'Flows in networks', Princeton University Press, Princeton, NJ (1962)

12. A. Frank \& A. Gyárfás: 'How to orient the edges of a graph?', Colloquia Mathematica Societatis János Bolyai 18, 353-364 (1976)

13. F. Harary, J. Krarup, and A. Schwenk: 'Graphs suppressible to an edge', Canadian Mathematical Bulletin 15, 201204 (1971)

14. J. Kára, J. Kratochvíl \& D. R. Wood: 'On the complexity of the balanced vertex ordering problem', Proceedings of COCOON2005, LNCS 3595, 849-858 (2005)

15. H.G. Landau: 'On dominance relations and the structure of animal societies III. The condition for a score structure' The Bulletin of Mathematical Biophysics 15, 143148 (1953)

16. D. Mubayi, T.G. Will \& D.B. West: 'Realizing Degree Imbalances in Directed Graphs', Discrete Mathematics 239(173), 147-153 (2001)

17. C. Nash-Williams: 'On orientations, connectivity and odd vertex pairings in finite graphs', Canadian Journal of Mathematics 12, 555-567 (1960)

18. H. Robbins: 'A theorem on graphs with an application to a problem of traffic control', American Mathematical Monthly 46, 281-283 (1939)

19. T. J. Schaefer: 'The complexity of satisfiability problems', Proceedings of the 10th Annual ACM Symposium on Theory of Computing, 216-226 (1978) 


\section{Appendix A Proof of Lemma 5}

Let $\varphi$ be a not-all-equal at most 3-SAT formula with $n$ variables $\left\{x_{1}, \cdots, x_{n}\right\}$ and $m$ clauses $\left\{c_{1}, \cdots, c_{m}\right\}$ and for all $i \in \llbracket 1, n \rrbracket$, let $k_{i} \in \mathbb{N}$ be the number of occurences of $x_{i}$ in $\varphi$. We assume that there is at least one variable $x_{i}$ that has at least 4 occurences in $\varphi$ (otherwise $\varphi$ is already a not-all-equal at most 3-SAT(3V) formula) and we will build from $\varphi$ a not-all-equal at most 3-SAT $(3 \mathrm{~V}) \varphi^{\prime}$ such that $\varphi$ and $\varphi^{\prime}$ are equisatisfiable as follows.

- For all $i \in \llbracket 1, n \rrbracket$, if $k_{i} \geq 4$ then we introduce $k_{i}$ new variables $\left\{x_{i}^{1}, \cdots, x_{i}^{k_{i}}\right\}$ and for $l \in \llbracket 1, k_{i} \rrbracket$ we replace the $l$-th occurence of $x_{i}$ in $\varphi$ with $x_{i}^{l}$.

- For all $i \in \llbracket 1, n \rrbracket$, if $k_{i} \geq 4$ then we add $k_{i}$ new clauses $\left\{c_{x_{i}}^{1}, \cdots, c_{x_{i}}^{k_{i}}\right\}$ where for $l \in \llbracket 1, k_{i}-1 \rrbracket, c_{x_{i}}^{l}=\left(x_{i}^{l} \vee \neg x_{i}^{l+1}\right)$ and $c_{x_{i}}^{l}=\left(x_{i}^{l} \vee \neg x_{i}^{1}\right)$.

Suppose there exists an assignment $v:\left\{x_{1}, \cdots, x_{n}\right\} \rightarrow\{$ TRUE, FALSE $\}$ of $x_{1}, \cdots, x_{n}$ satisfying $\varphi$. Then

$$
\begin{aligned}
& v^{\prime}: x_{i} \mapsto v\left(x_{i}\right) \quad \forall i \in \llbracket 1, n \rrbracket \text { s.t. } k_{i} \leq 3 ; \\
& x_{i}^{l} \mapsto v\left(x_{i}\right) \quad \forall i \in \llbracket 1, n \rrbracket \text { s.t. } k_{i} \geq 4 \text { and } \forall l \in \llbracket 1, k_{i} \rrbracket ;
\end{aligned}
$$

is an assignment of the variables $x_{i}$ and $x_{i}^{l}$ satisfying $\varphi^{\prime}$ for

- $\forall j \in \llbracket 1, m \rrbracket$, the values of the literals of $c_{j}$ w.r.t. $v$ and $v^{\prime}$ are piecewise equal so $v^{\prime}\left(c_{j}\right)=v\left(c_{j}\right)=$ TRUE and $v^{\prime}$ is not-all-equal for $c_{j}$ as well as $v$ is;

- $\forall i \in \llbracket 1, n \rrbracket$ s.t. $k_{i} \geq 4, \forall l \in \llbracket 1, k_{i}-1 \rrbracket, v^{\prime}\left(x_{i}^{l}\right)=v^{\prime}\left(x_{i}^{l+1}\right)=v\left(x_{i}\right)$ and $v^{\prime}\left(x_{i}^{k_{i}}\right)=v^{\prime}\left(x_{i}^{1}\right)=$ $v\left(x_{i}\right)$ so we directly have $\forall l \in \llbracket 1, k_{i}-1 \rrbracket, v^{\prime}\left(c_{x_{i}}^{l}\right)=$ TRUE and $v^{\prime}\left(c_{x_{i}}^{k_{i}}\right)=$ TRUE and $v^{\prime}$ is not-all-equal for each of these clauses since they all consist of two literals having opposite values w.r.t. $v^{\prime}$.

As an example, for a formula

$$
\varphi=\left(x_{1} \vee \neg x_{2} \vee x_{3}\right) \wedge\left(\neg x_{1} \vee \neg x_{3} \vee x_{4}\right) \wedge\left(x_{1} \vee \neg x_{2}\right) \wedge\left(\neg x_{1} \vee \neg x_{3} \vee \neg x_{4}\right) \wedge\left(x_{1} \vee x_{3}\right),
$$

where $x_{1}$ occurs five times and $x_{3}$ four so we add nine new variables $x_{1}^{1}, x_{1}^{2}, x_{1}^{3}, x_{1}^{4}, x_{1}^{5}$, $x_{3}^{1}, x_{3}^{2}, x_{3}^{3}$ and $x_{3}^{4}$ and nine new clauses:

$$
\begin{aligned}
\varphi^{\prime}= & \left(x_{1}^{1} \vee \neg x_{2} \vee x_{3}^{1}\right) \wedge\left(\neg x_{1}^{2} \vee \neg x_{3}^{2} \vee x_{4}\right) \wedge\left(x_{1}^{3} \vee \neg x_{2}\right) \wedge\left(\neg x_{1}^{4} \vee \neg x_{3}^{3} \vee \neg x_{4}\right) \wedge\left(x_{1}^{5} \vee x_{3}^{4}\right) \\
& \wedge\left(x_{1}^{1} \vee \neg x_{1}^{2}\right) \wedge\left(x_{1}^{2} \vee \neg x_{1}^{3}\right) \wedge\left(x_{1}^{3} \vee \neg x_{1}^{4}\right) \wedge\left(x_{1}^{4} \vee \neg x_{1}^{5}\right) \wedge\left(x_{1}^{5} \vee \neg x_{1}^{1}\right) \\
& \wedge\left(x_{3}^{1} \vee \neg x_{3}^{2}\right) \wedge\left(x_{3}^{2} \vee \neg x_{3}^{3}\right) \wedge\left(x_{3}^{3} \vee \neg x_{3}^{4}\right) \wedge\left(x_{3}^{4} \vee \neg x_{3}^{1}\right)
\end{aligned}
$$

Now suppose there exists an assignment $v^{\prime}$ of the $x_{i}$ and $x_{i}^{l}$ satisfying $\varphi^{\prime}$ and let $i \in \llbracket 1, n \rrbracket$ such that $k_{i} \geq 4$. If we take a look at the clauses $c_{x_{i}}^{1}, \cdots, c_{x_{i}}^{k_{i}}$, we notice that if $v^{\prime}\left(x_{i}^{1}\right)=$ FALSE then for $c_{x_{i}}^{1}$ to be satisfied, $v^{\prime}\left(\neg x_{i}^{2}\right)=$ TRUE, i.e. $v^{\prime}\left(x_{i}^{2}\right)=$ FALSE, then for $c_{x_{i}}^{2}$ to be satisfied, $v^{\prime}\left(\neg x_{i}^{3}\right)=$ TRUE ...etc. Repeating this argument, we obtain that if $v^{\prime}\left(x_{i}^{1}\right)=$ FALSE then $v^{\prime}\left(x_{i}^{1}\right)=v^{\prime}\left(x_{i}^{2}\right)=\cdots=v^{\prime}\left(x_{i}^{k_{i}}\right)=$ FALSE. Similarly, if $v^{\prime}\left(x_{i}^{k_{i}}\right)=$ TRUE then 
for $c_{x_{i}}^{k_{i}}$ to be satisfied, $v^{\prime}\left(\neg x_{i}^{k_{i}-1}\right)=$ FALSE, i.e. $v^{\prime}\left(x_{i}^{k_{i}-1}\right)=$ TRUE, then for $c_{x_{i}}^{k_{i}-1}$ to be satisfied, $v^{\prime}\left(\neg x_{i}^{k_{i}-2}\right)=$ FALSE ...etc. Hence if $v^{\prime}\left(x_{i}^{k_{i}}\right)=$ TRUE then $v^{\prime}\left(x_{i}^{k_{i}}\right)=v^{\prime}\left(x_{i}^{k_{i}-1}\right)=$ $\cdots=v^{\prime}\left(x_{i}^{1}\right)=$ TRUE. This yields that

$$
\forall i \in \llbracket 1, n \rrbracket \text { s.t. } k_{i} \geq 4, v^{\prime}\left(x_{i}^{1}\right)=v^{\prime}\left(x_{i}^{2}\right)=\cdots=v^{\prime}\left(x_{i}^{k_{i}}\right) .
$$

Hence for all $i \in \llbracket 1, n \rrbracket$ such that $k_{i} \geq 4$, we can replace $x_{i}^{1}, \cdots, x_{i}^{k_{i}}$ by a unique variable $x_{i}$ and doing so the clauses $c_{x_{i}}^{1}, \cdots, c_{x_{i}}^{k_{i}}$ become trivial and can be removed and only $\varphi$ remains. So the following assignment of $x_{1}, \cdots, x_{n}$ :

$$
v: \begin{array}{ll}
x_{i} \mapsto v^{\prime}\left(x_{i}\right) & \forall i \in \llbracket 1, n \rrbracket \text { s.t. } k_{i} \leq 3 ; \\
x_{i} \mapsto v^{\prime}\left(x_{i}^{1}\right) \quad \forall i \in \llbracket 1, n \rrbracket \text { s.t. } k_{i} \geq 4 ;
\end{array}
$$

satisfies $\varphi$. We have just shown that $\varphi$ and $\varphi^{\prime}$ are equisatisfiable. 